Recent results on strangeness, charm and beauty production at HERA

This article has been downloaded from IOPscience. Please scroll down to see the full text article.

2010 Chinese Phys. C 34757

(http://iopscience.iop.org/1674-1137/34/6/025)

View the table of contents for this issue, or go to the journal homepage for more

Download details:

IP Address: 131.169.42.235

The article was downloaded on 15/03/2011 at 07:14

Please note that terms and conditions apply. 


\title{
Recent results on strangeness, charm and beauty production at HERA
}

\author{
FANG Shuang-Shi(房双世) $)^{1)}$ (on behalf of the H1 and ZEUS collaborations) \\ Deutsches Elektronen-Synchrotron, Hamburg, 22607, Germany
}

\begin{abstract}
Based on the data collected with the H1 and ZEUS detectors at HERA, recent results on strangeness, charm and beauty production in ep collision are presented.
\end{abstract}

Key words strange hadrons, heavy flavor production, ep collision

PACS 13.60.-r, 14.40.Df, 14.40.Lb

\section{Introduction}

At HERA strange quarks are produced in the nonperturbative process of color string fragmentation, which constitutes the dominant production mechanism of strange hadrons. In deep-inelastic scattering (DIS), strange quarks also originate from the strange sea in the nucleon, boson-gluon fusion and heavy quark decays. Therefore the study of strange hadrons production allows us to investigate the strong interaction in both the perturbative and non-perturbative regimes. In addition data on the $\Lambda-\bar{\Lambda}$ production asymmetry from HERA are of interest as an experimental constraint for theories of baryon number transfer [1].

Due to the large mass scale, heavy quark production offers a sensitive test of QCD predictions, which is dominated by boson-gluon fusion where the photon is emitted from the incoming lepton and the gluon from the proton. The large charm production cross section offers a unique place to study excited charm and charm-strange mesons produced in ep collisions. The further measurements of these states will help us understand their properties which challenge the theoretical description of heavy quark resonances.

Compared to charm production, beauty production is suppressed by a factor of approximately 200. Charm and beauty measurements performed at HERA so far relied on the tagging of only one heavy quark in each event. While the charm measurements were mostly based on the reconstruction of D mesons, in beauty measurements different experimental techniques, semileptonic decays or lifetime signatures or both, can be used to tag a beauty quark.

In this talk, we present the recent results on strangeness, charm and beauty production based on the data collected with the ZEUS and H1 detectors. The ZEUS and H1 detectors are two multipurpose magnetic detector designed to study ep scattering at HERA which are described in detail in Ref. [2].

\section{Strangeness production}

\section{$2.1 \mathrm{~K}_{\mathrm{S}}^{0}, \Lambda(\bar{\Lambda})$ and $\mathrm{K}^{* \pm}$ production}

The data collected by the H1 detector corresponding to an integrated luminosity of $50 \mathrm{pb}^{-1}$ was used in this analysis [3]. The measurement was performed in the DIS regime with the energy requirement of the scattered electron, $E_{\mathrm{e}^{\prime}}>11 \mathrm{GeV}$. The virtuality of the exchanged photon is required to be $2<Q^{2}<$ $100 \mathrm{GeV}^{2}$ for $\mathrm{K}_{\mathrm{S}}^{0}$ and $\Lambda(\bar{\Lambda})$, and $5<Q^{2}<100 \mathrm{GeV}^{2}$ for $\mathrm{K}^{* \pm}$.

The selection of strange mesons depends on the measurement of their final charged daughter particles. The tracks of the daughters of $\mathrm{K}_{\mathrm{S}}^{0}$ and $\Lambda(\bar{\Lambda})$ must be in the central region of the detector and must point back to a common secondary decay vertex with the direction of flight of the mother particle constrained to the primary event vertex. The transverse momentum $p_{\mathrm{T}}$ and the pseudorapidity $\eta$ of the strange me-

Received 25 January 2010

1)E-mail: shuangshi.fang@desy.de

(C)2009 Chinese Physical Society and the Institute of High Energy Physics of the Chinese Academy of Sciences and the Institute of Modern Physics of the Chinese Academy of Sciences and IOP Publishing Ltd 
son candidates must satisfy $0.5<p_{\mathrm{T}}<3.5 \mathrm{GeV}$ and $|\eta|<1.3$ for $\mathrm{K}_{\mathrm{S}}^{0}$ and $\Lambda(\bar{\Lambda})$, and $p_{\mathrm{T}}>1.0 \mathrm{GeV}$ and $|\eta|<1.5$ for $\mathrm{K}^{* \pm}$.

After above selection, the number of signals, about $213000 \mathrm{~K}_{\mathrm{S}}^{0}, 22000 \Lambda, 20000 \bar{\Lambda}$ and $79000 \mathrm{~K}^{* \pm}$, is obtained by fitting the invariant mass spectra with two Gaussian plus a background functions. The total inclusive production cross sections in the visible kinematic range are determined to be:

$$
\begin{aligned}
& \left.\sigma_{\text {vis }}\left(\mathrm{ep} \rightarrow \mathrm{eK}_{\mathrm{S}}^{0} \mathrm{X}\right)=21.18 \pm 0.09 \text { (stat.) }\right)_{-1.23}^{+1.19} \text { (syst.) nb, } \\
& \sigma_{\text {vis }}(\mathrm{ep} \rightarrow \mathrm{e} \Lambda \mathrm{X})=3.96 \pm 0.06 \text { (stat.) }{ }_{-0.24}^{+0.23} \text { (syst.) nb, } \\
& \sigma_{\text {vis }}(\mathrm{ep} \rightarrow \mathrm{e} \bar{\Lambda} \mathrm{X})=3.94 \pm 0.07 \text { (stat.) }{ }_{-0.24}^{+0.23} \text { (syst.) nb, } \\
& \sigma_{\text {vis }}\left(\mathrm{ep} \rightarrow \mathrm{eK}^{* \pm} \mathrm{X}\right)=7.36 \pm 0.09 \text { (stat.) } \pm 0.88 \text { (syst.) nb, }
\end{aligned}
$$

all of them are consistent with Monte Carlo (MC) expectations.

$\mathrm{K}_{\mathrm{S}}^{0}, \Lambda$ and $\mathrm{K}^{* \pm}$ cross sections are also measured differentially in laboratory frame as functions of the kinematic variable $Q^{2}$, the pseudorapidity $\eta$, trans- verse momentum $p_{\mathrm{T}}$. The differential cross sections for $\mathrm{K}_{\mathrm{S}}^{0}$ in the laboratory frame as a function of $Q^{2}$, $p_{\mathrm{T}}$ and $\eta$ are shown in Fig. 1 (see Ref. [3] for more figures). The differential cross section measurements quickly decrease when the value of the variables $Q^{2}$ and $p_{\mathrm{T}}$ increase. The $\eta$ cross section is flat for $\mathrm{K}_{\mathrm{S}}^{0}$ but rises in the forward region for $\Lambda$. The CDM model with $\lambda_{s}=0.3$ agrees with the data although it has difficulties with the $\eta$ shape and at low $p_{\mathrm{T}}$. The $\mathrm{K}^{* \pm}$ differential cross section measured in the laboratory frame are also compared to models with $\lambda_{s}=0.286$. As was observed for $K_{\mathrm{S}}^{0}$ and $\Lambda$, for $K^{* \pm}$ both models fail to describe the $\eta$ cross section but in general CDM seems better than MEPS.

The comparison to CDM and MEPS models shows that no single model prediction or strangeness factor is able to describe all results.

The measurements of the $\Lambda$ and $\bar{\Lambda}$ cross sections show that there is no evidence of baryon number transfer from the proton beam.
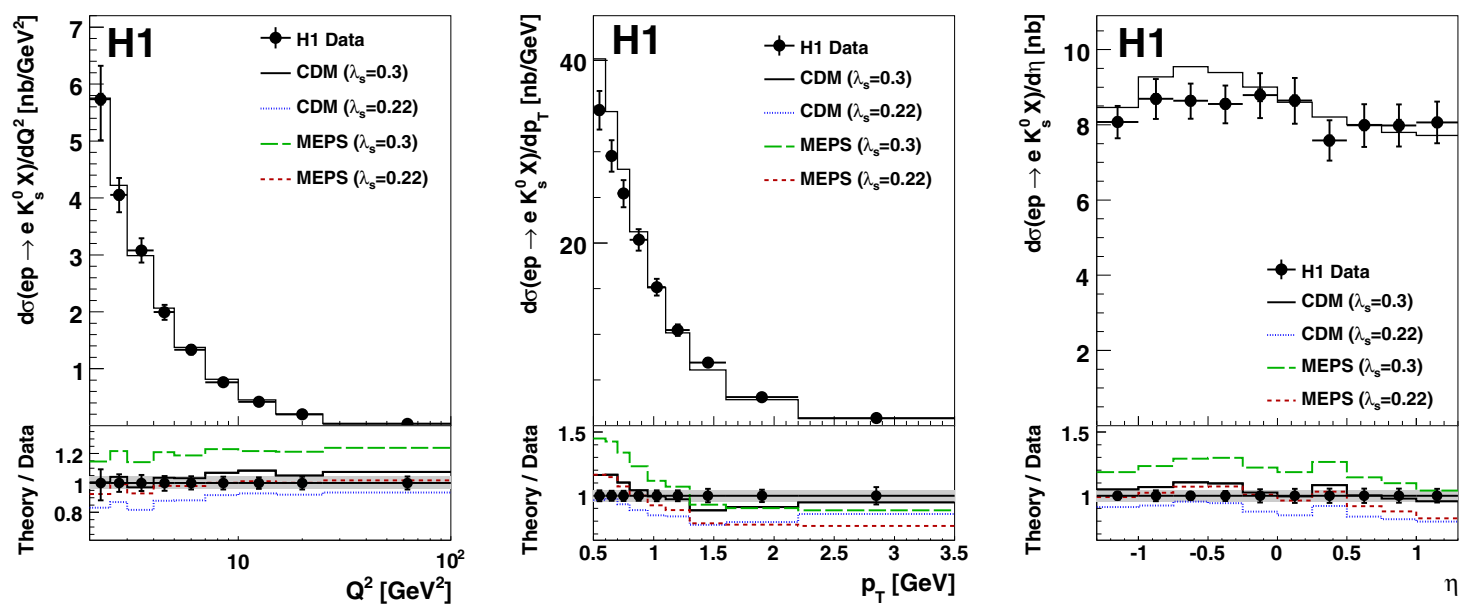

Fig. 1. Differential production cross sections for $\mathrm{K}_{\mathrm{S}}^{0}$ in the laboratory frame as a function of $Q^{2}, p_{\mathrm{T}}$ and $\eta$.
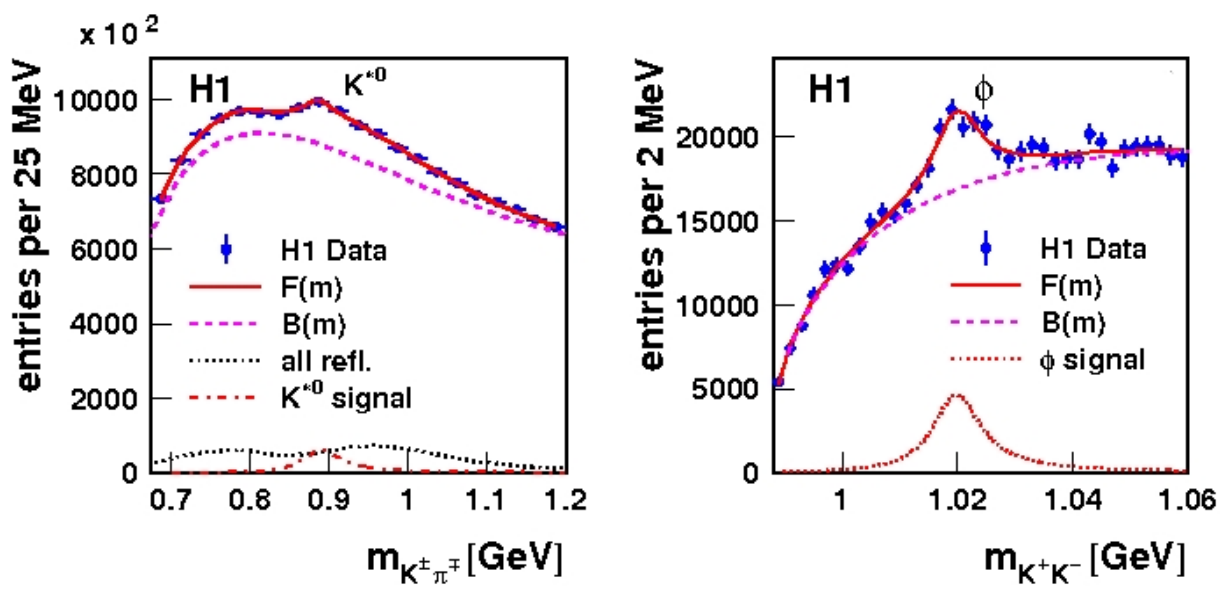

Fig. 2. The invariant mass spectra for $\mathrm{K}^{ \pm} \pi^{\mp}$ and for $\mathrm{K}^{+} \mathrm{K}^{-}$. The full curves show the result of the fit; the dashed curves correspond to the contribution of the combinatorial background. 


\section{$2.2 \quad \mathrm{~K}^{* 0}$ and $\phi$ production}

Based on the data, an integrated luminosity of $50 \mathrm{pb}^{-1}$, recorded with the $\mathrm{H} 1$ detector. This analysis [4] was performed in photoproduction with the requirement of $Q^{2}<1 \mathrm{GeV}^{2}$. The reconstructed $\gamma \mathrm{p}$ center-of-mass energy is required in the range $174<W<256 \mathrm{GeV}$, which corresponds to an average $\gamma$ p center-of-mass energy of $\langle W\rangle=210 \mathrm{GeV}$.

The mesons are reconstructed using $\mathrm{K}^{* 0} \rightarrow \mathrm{K}^{+} \pi^{-}$ and $\phi \rightarrow \mathrm{K}^{+} \mathrm{K}^{-}$decays. The requirements on the charged tracks reconstructed in CJCs are $p_{\mathrm{T}}>$ $0.15 \mathrm{GeV}$ and $|\eta|<1.5$. The meson candidates must satisfy $0.5<p_{\mathrm{T}}<7 \mathrm{GeV}$ and $|\eta|<1$. Clear $\mathrm{K}^{* 0}$ and $\phi$ signals are observed in the invariant mass distribution of $\mathrm{K}^{ \pm} \pi^{\mp}$ and $\mathrm{K}^{+} \mathrm{K}^{-}$shown in Fig. 2 .

After considering the acceptance correction obtained from the MC simulation, the inclusive nondiffractive photoproduction cross sections for $\mathrm{K}^{* 0}$ and $\phi$ mesons are found to be:

$\sigma_{\text {vis }}\left(\gamma \mathrm{p} \rightarrow \mathrm{K}^{* 0} \mathrm{X}\right)=6260 \pm 350$ (stat.) \pm 860 (syst.) nb, $\sigma_{v i s}(\gamma \mathrm{p} \rightarrow \phi \mathrm{X})=2400 \pm 180$ (stat.) \pm 340 (syst.) nb.

\subsection{Inclusive $\mathrm{K}_{\mathrm{S}}^{0} \mathrm{~K}_{\mathrm{S}}^{0}$ resonances}

The data used in this analysis [5] corresponds to the full HERA luminosity of $0.5 \mathrm{fb}^{-1}$ taken with the ZEUS detector. The $\mathrm{K}_{\mathrm{S}}^{0}$ meson was identified via its charged-decay mode, $\mathrm{K}_{\mathrm{S}}^{0} \rightarrow \pi^{+} \pi^{-}$. Both tracks from the same secondary decay vertex were assigned the mass of the charged pion and invariant mass of each charged pion pair was calculated. Three enhance- ments, $\mathrm{f}_{2}(1270) / \mathrm{a}_{2}^{0}(1320), \mathrm{f}_{2}^{\prime}(1525)$ and $\mathrm{f}_{0}(1710)$, are clearly observed over a large background in the invariant mass of $K_{\mathrm{S}}^{0} \mathrm{~K}_{\mathrm{S}}^{0}$ shown in Fig. 3. Their widths and masses from two different fits, considering interference or not, are shown in Table 1, which are consistent with the PDG values [6] for each resonance.

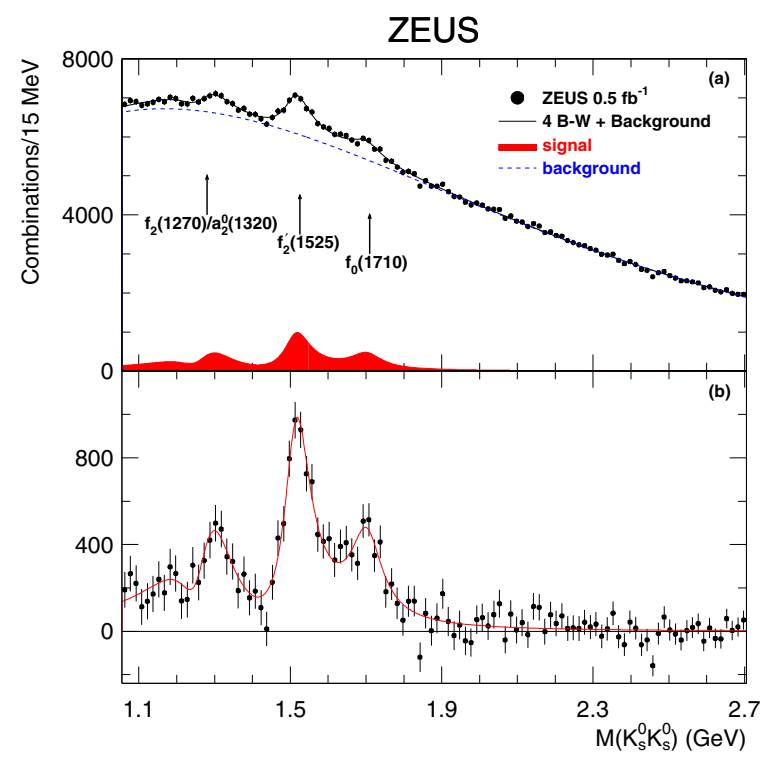

Fig. 3. (a) The measured $K_{\mathrm{S}}^{0} K_{\mathrm{S}}^{0}$ invariant-mass spectrum (dots). The solid line is the result of the fit and the dashed line represents the background function. (b) Background-subtracted $\mathrm{K}_{\mathrm{S}}^{0} \mathrm{~K}_{\mathrm{S}}^{0}$ invariant mass spectrum (dots); the result of the fit is shown as a solid line.

Table 1. The $\mathrm{K}_{\mathrm{S}}^{0} \mathrm{~K}_{\mathrm{S}}^{0}$ mass spectrum fitting results.

\begin{tabular}{lcccc}
\hline fit & \multicolumn{2}{c}{ no interference } & \multicolumn{2}{c}{ interference } \\
\cline { 2 - 5 } in $\mathrm{MeV}$ & mass & width & mass & width \\
\hline $\mathrm{f}_{2}(1270)$ & $1304 \pm 6$ & $61 \pm 11$ & $1268 \pm 10$ & $176 \pm 17$ \\
$\mathrm{a}_{2}^{0}(1320)$ & & $71 \pm 5_{-2}^{+17}$ & $1512 \pm 3_{-0.5}^{+1.4}$ & $114 \pm 14$ \\
$\mathrm{f}_{2}^{\prime}(1525)$ & $1523 \pm 3_{-8}^{+2}$ & $125 \pm 12_{-32}^{+19}$ & $1701 \pm 5_{-2}^{+9}$ & $83 \pm 9_{-4}^{+5}$ \\
$\mathrm{f}_{0}(1710)$ & $1692 \pm 6_{-3}^{+9}$ & & & $100 \pm 24_{-22}^{+7}$ \\
\hline
\end{tabular}
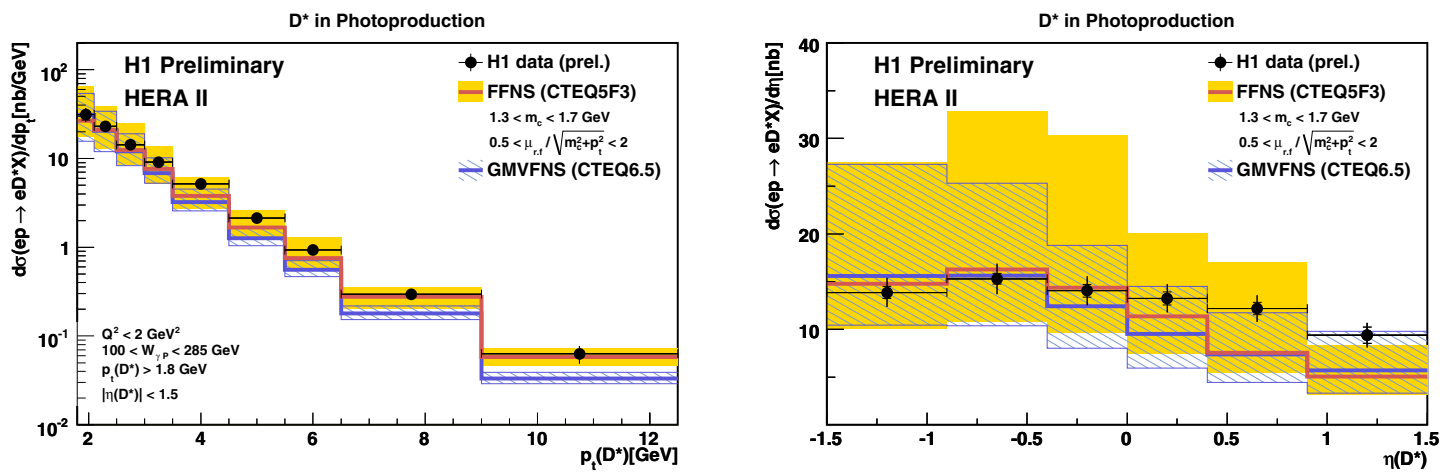

Fig. 4. Differential cross section as a function of $p_{\mathrm{T}}\left(\mathrm{D}^{*}\right)$ and $\eta\left(\mathrm{D}^{*}\right)$. 


\section{Charm production}

\section{1 $\mathrm{D}^{*}$ mesons in photoproduction}

The data used in this analysis [7] was collected with the $\mathrm{H} 1$ detector and corresponds to an integrated luminosity of $93 \mathrm{pb}^{-1}$. This analysis covers a kinematic region of small photon virtuality $Q^{2}<2 \mathrm{GeV}^{2}$ and photon-proton center-of-mass energy of $100<W<285 \mathrm{GeV}$. The $\mathrm{D}^{*}$ meson reconstructed from the decay chain $\mathrm{D}^{*} \rightarrow \mathrm{D}^{0} \pi_{\mathrm{s}} \rightarrow(\mathrm{K} \pi) \pi_{\mathrm{s}}$ was required to satisfy $p_{\mathrm{T}}>1.8 \mathrm{GeV}$ and $|\eta|<1.5$.

The single differential cross-sections and the comparison to the NLO QCD calculation are given for $p_{\mathrm{T}}\left(\mathrm{D}^{*}\right)$ and $\eta\left(\mathrm{D}^{*}\right)$, which are shown in Fig. 4 . In general the NLO QCD calculation given in FMNR programme provides a reasonable description of data.

\subsection{Excited charm and charm-strange pro- duction}

The production of excited charm charm-strange mesons, $\quad \mathrm{D}_{1}(2420)^{0}, \quad \mathrm{D}_{2}^{*}(2460)^{0}$ and $\mathrm{D}_{\mathrm{s} 1}(2536)^{ \pm}$, mesons was investigated in both photoproduction and DIS regime [8] using an integrated luminosity of $126 \mathrm{pb}^{-1}$. Two $\mathrm{D}^{0}$ decay modes, $\mathrm{D}^{0} \rightarrow \mathrm{K}^{-} \pi^{+}$and $\mathrm{D}^{0} \rightarrow \mathrm{K}^{-} \pi^{+} \pi^{+} \pi^{-}$, are used to reconstruct $\mathrm{D}^{* \pm}$ meson from its golden decay mode $\mathrm{D}^{* \pm} \rightarrow \mathrm{D}^{0} \pi_{\mathrm{s}}^{ \pm}$, where $\pi_{\mathrm{s}}$ is referred to the "soft" pion in the $\mathrm{D}^{* \pm}$ decays.

To reconstruct the $\mathrm{D}_{1}(2420)^{0}, \mathrm{D}_{2}^{*}(2460)^{0} \rightarrow$ $D^{* \pm} \pi^{\mp}$, an excited charm meson candidate was formed by combining each selected $\mathrm{D}^{* \pm}$ candidate with an additional track, assumed to be a pion $\left(\pi_{\mathrm{a}}\right)$, with a charge opposite to that of the $\mathrm{D}^{* \pm}$ candidate. Fig. 5a shows the $M\left(\mathrm{D}^{* \pm} \pi_{\mathrm{a}}^{\mp}\right)$ distribution for $\mathrm{D}^{* \pm}$ meson candidates reconstructed in both decay channels. A clear enhancement is seen in the range $2.4<M\left(\mathrm{D}^{* \pm} \pi_{\mathrm{a}}^{\mp}\right)<2.5 \mathrm{GeV}$, where contributions from $\mathrm{D}_{1}(2420)^{0}$ and $\mathrm{D}_{2}^{*}(2460)^{0}$ mesons are expected. The wide $\mathrm{D}_{1}(2430)^{0}$ meson, which is also expected to contribute to $M\left(\mathrm{D}^{* \pm} \pi_{\mathrm{a}}^{\mp}\right)$ distribution, is not distinguishable from background due to its large width. No enhancement is observed in the $M\left(\mathrm{D}^{* \pm} \pi_{\mathrm{a}}^{\mp}\right)$ distribution for wrong charge combinations formed by combining a $\mathrm{D}^{* \pm}$ candidate and $\pi_{\mathrm{a}}$ with the same charge.

The decay $\mathrm{D}_{2}^{*}(2460)^{0} \rightarrow \mathrm{D}^{ \pm} \pi^{\mp}$ was also used to reconstruct $\mathrm{D}_{2}^{*}(2460)^{0}$ in this analysis. The candidate was formed by combining each selected $\mathrm{D}^{ \pm}$candidate with an additional track, assumed to be a pion $\left(\pi_{\mathrm{a}}\right)$, with a charge opposite to that of the $\mathrm{D}^{ \pm}$. A small excess is seen around the nominal mass of the $\mathrm{D}_{2}^{*}(2460)^{0}$ meson in the distribution of $M\left(\mathrm{D}^{ \pm} \pi_{\mathrm{a}}^{\mp}\right)$ shown in Fig. 5(b). As expected from parity and the angular momentum conservation for a $1^{+}$state, no indication of $\mathrm{D}_{1}(2420)^{0}$ decaying into $\mathrm{D}^{ \pm} \pi^{\mp}$ is observed.
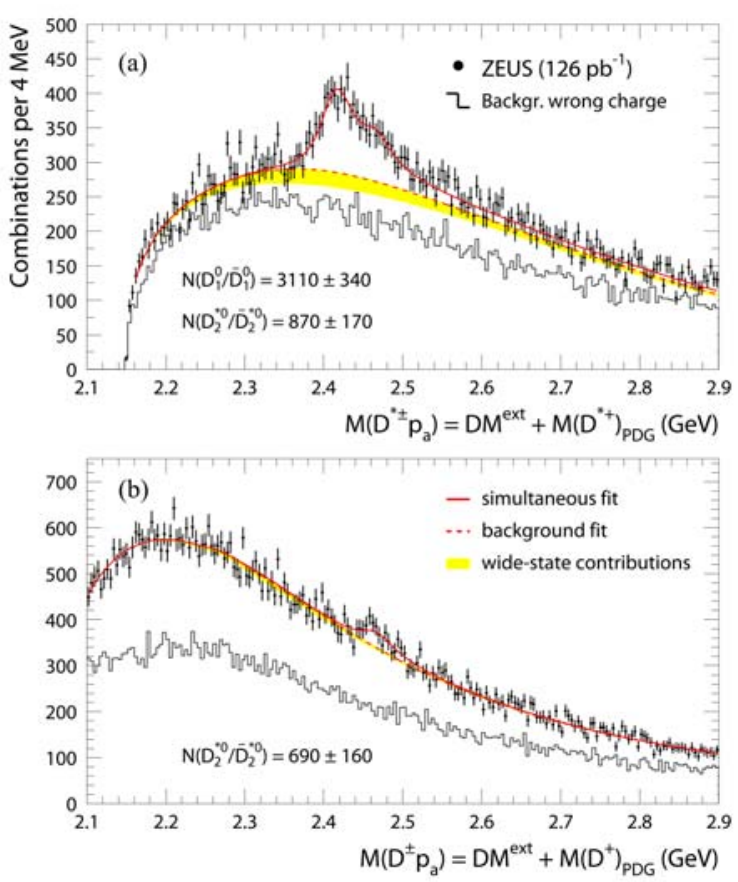

Fig. 5. The distributions of $M\left(\mathrm{D}^{* \pm} \pi_{\mathrm{a}}^{\mp}\right)$ and $M\left(\mathrm{D}^{ \pm} \pi_{\mathrm{a}}^{\mp}\right)$.
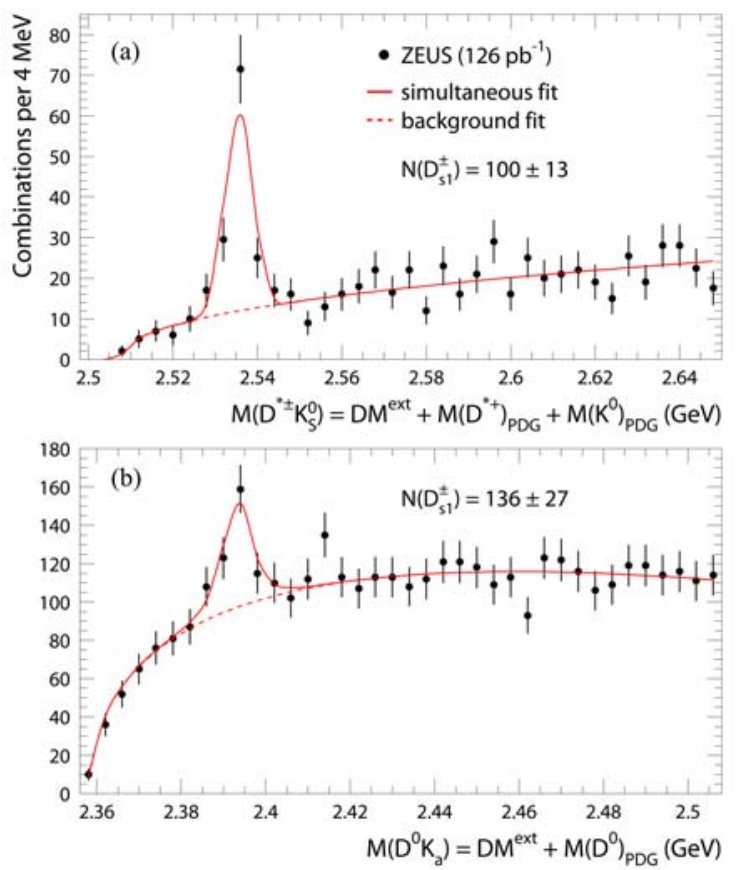

Fig. 6. The distributions of $M\left(\mathrm{D}^{* \pm} \mathrm{K}_{\mathrm{S}}^{0}\right)$ and $M\left(\mathrm{D}^{0} \mathrm{~K}_{\mathrm{a}}^{ \pm}\right)$.

The $\mathrm{D}_{\mathrm{s} 1}(2536)^{ \pm}$was detected in two decay modes, $\mathrm{D}_{\mathrm{s} 1}(2536)^{ \pm} \rightarrow \mathrm{D}^{* \pm} \mathrm{K}_{\mathrm{S}}^{0}$ and $\mathrm{D}_{\mathrm{s} 1}(2536)^{ \pm} \rightarrow \mathrm{D}^{0} \mathrm{~K}^{ \pm}$. For 
the first decay mode, the candidate was formed by combining each selected $\mathrm{D}^{* \pm}$ candidate with the $\mathrm{K}_{\mathrm{S}}^{0}$ candidates reconstructed in the same event. For the second decay mode, the candidate was formed by combining each selected untagged $\mathrm{D}^{0}$ candidate with an additional track, assumed to be a kaon $\left(K_{a}\right)$, with a charge opposite to that of particle taken as a kaon to form the $\mathrm{D}^{0}$ candidate. The $M\left(\mathrm{D}^{* \pm} \mathrm{K}_{\mathrm{S}}^{0}\right)$ and $M\left(\mathrm{D}^{0} \mathrm{~K}_{\mathrm{a}}^{ \pm}\right)$distributions are shown in Fig. 6(a) and Fig. 6(b), respectively. Clear signal are observed around the mass of $\mathrm{D}_{\mathrm{s} 1}(2536)^{ \pm}$.

The fragmentation fractions of c quarks hadronising into a specific charm mesons obtained from ZEUS, OPAL and ALEPH, as well as the predictions of the thermodynamical model, are listed in Table 2. The results from ZEUS are consistent with those obtained in $\mathrm{e}^{+} \mathrm{e}^{-}$annihilations, while $\mathrm{f}\left(\mathrm{c} \rightarrow \mathrm{D}_{1}^{0}\right)$ and $\mathrm{f}\left(\mathrm{c} \rightarrow \mathrm{D}_{2}^{* 0}\right)$ are above the predictions of the thermodynamical model.

Table 2. The fragmentation fractions of $\mathrm{c}$ quarks hadronising into the $\mathrm{D}_{1}^{0}, \mathrm{D}_{2}^{* 0}$ and $\mathrm{D}_{\mathrm{s} 1}^{+}$ $\operatorname{mesons}(\%)$.

\begin{tabular}{cccc}
\hline & $\mathrm{f}\left(\mathrm{c} \rightarrow \mathrm{D}_{1}^{0}\right)$ & $\mathrm{f}\left(\mathrm{c} \rightarrow \mathrm{D}_{2}^{* 0}\right)$ & $\mathrm{f}\left(\mathrm{c} \rightarrow \mathrm{D}_{\mathrm{s} 1}^{+}\right)$ \\
\hline ZEUS & $3.5 \pm 0.4_{-0.6}^{+0.4}$ & $3.8 \pm 0.7_{-0.6}^{+0.5}$ & $1.11 \pm 0.16_{-0.10}^{+0.08}$ \\
OPAL [9] & $2.1 \pm 0.7 \pm 0.3$ & $5.2 \pm 2.2 \pm 1.3$ & $1.6 \pm 0.4 \pm 0.3$ \\
ALEPH [10] & & & $0.94 \pm 0.22 \pm 0.07$ \\
Model [11] & 1.7 & 2.4 & 0.54 \\
\hline
\end{tabular}

To search for the $\mathrm{D}^{* \prime}(2460) \rightarrow \mathrm{D}^{* \pm} \pi^{+} \pi^{-}$decays, a $\mathrm{D}^{* \prime}(2460)$ candidate was formed by combining each selected $\mathrm{D}^{* \pm}$ with two additional tracks with opposite charges. No radially excited charm meson was observed in the mass spectrum of $\mathrm{D}^{* \pm} \pi^{+} \pi^{-}$, the upper limit $0.4 \%$ at $95 \%$ confidence level is given, which is stronger than the $0.9 \%$ obtained by OPAL Collaboration [12].

\section{Beauty production}

\subsection{Beauty in dijet photoproduction using electrons}

This analysis [13] was performed by the ZEUS collaboration in which the b quarks were tagged via semileptonic decays into electrons. The events with at least 2 jets with $E_{\mathrm{T}}^{\text {Jet1(2) }}>7(6) \mathrm{GeV}$ and $\left|\eta^{\text {Jet1(2) }}\right|<$ 2.5 were selected. Five discriminating variables, $\mathrm{d} E / \mathrm{d} x, p_{\mathrm{T}}^{\text {rel }}, E_{\mathrm{EMC}} / E_{\mathrm{CAL}}, \Delta \phi$ and $E_{\mathrm{CAL}} / p_{\text {track }}$, sensitive to electron identification as well as to semileptonic decays, were combined in a likelihood fit to extract the fractions of events containing $b$ quarks and those containing c quarks.

Differential cross sections as a function of $p_{\mathrm{T}}^{\mathrm{e}}$ and $\eta^{\mathrm{e}}$ are shown in Fig. 7. The measurements are in agreement with PYTHIA LO+PS MC predictions scaled according to the fit and the NLO QCD predictions calculated with the FMNR programme.

\section{ZEUS}
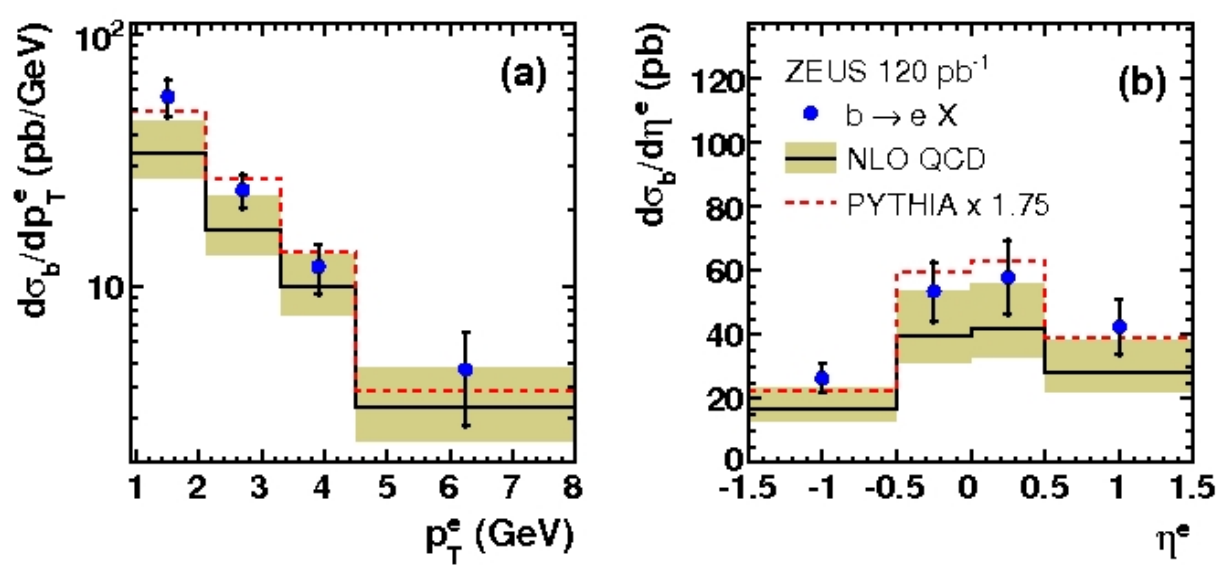

Fig. 7. Differential cross sections as a function of $p_{\mathrm{T}}^{\mathrm{e}}$ and $\eta^{\mathrm{e}}$.

\subsection{Beauty in dijet photoproduction using muons}

Two very similar analyses were performed by the H1 and ZEUS collaborations [14, 15] using semilep- tonic muons in the final state to tag $b$ quarks. In both analyses, photoproduction events with at least 2 jets with $E^{\mathrm{Jet1}(2)} T>7(6) \mathrm{GeV}$ were selected. The muon candidates were reconstructed in the region $-0.55<\eta^{\mu}<1.1$ (H1) and $-1.6<\eta^{\mu}<1.3$ (ZEUS) 
and had to have a transverse momentum $p_{\mathrm{T}}^{\mu}>2.5$ $\mathrm{GeV}(\mathrm{H} 1)$ and $p_{\mathrm{T}}^{\mu}>1.5 \mathrm{GeV}$ (ZEUS). The fraction of events containing b quarks was determined from a simultaneous fit of two discriminating variables, $p_{\mathrm{T}}^{\text {rel }}$ and the impact parameter $\delta$, where $\delta$ is defined as the point of closest approach in $x-y$ of the track helix of the muon candidate with respect to the interaction point.
Differential cross sections as a function of different kinematic variables are shown in Fig. 8 (ZEUS) and Fig. 9 (H1), respectively. Good agreement between data and NLO QCD predictions is observed, while the PYTHIA MC aslo describes the shapes well. In addition the differential cross section as a function of $\eta^{\mu}$ was compared to previous measurements [16, 17]. The results are found to be in very good agreement.

ZEUS
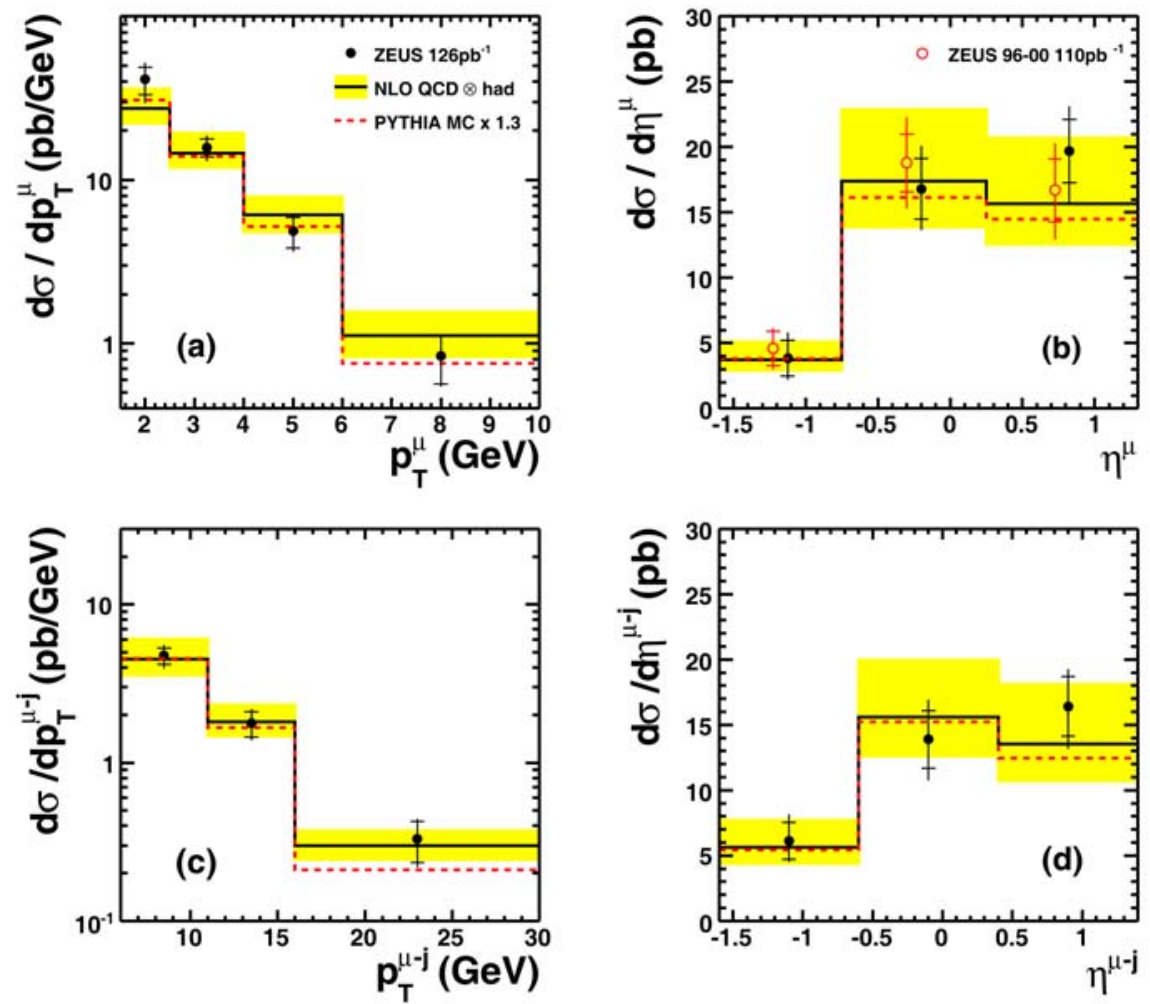

Fig. 8. Differential cross sections as a function of $p_{\mathrm{T}}^{\mu}, \eta^{\mu}, p_{\mathrm{T}}^{\mu-j}$ and $\eta^{\mu-j}$.
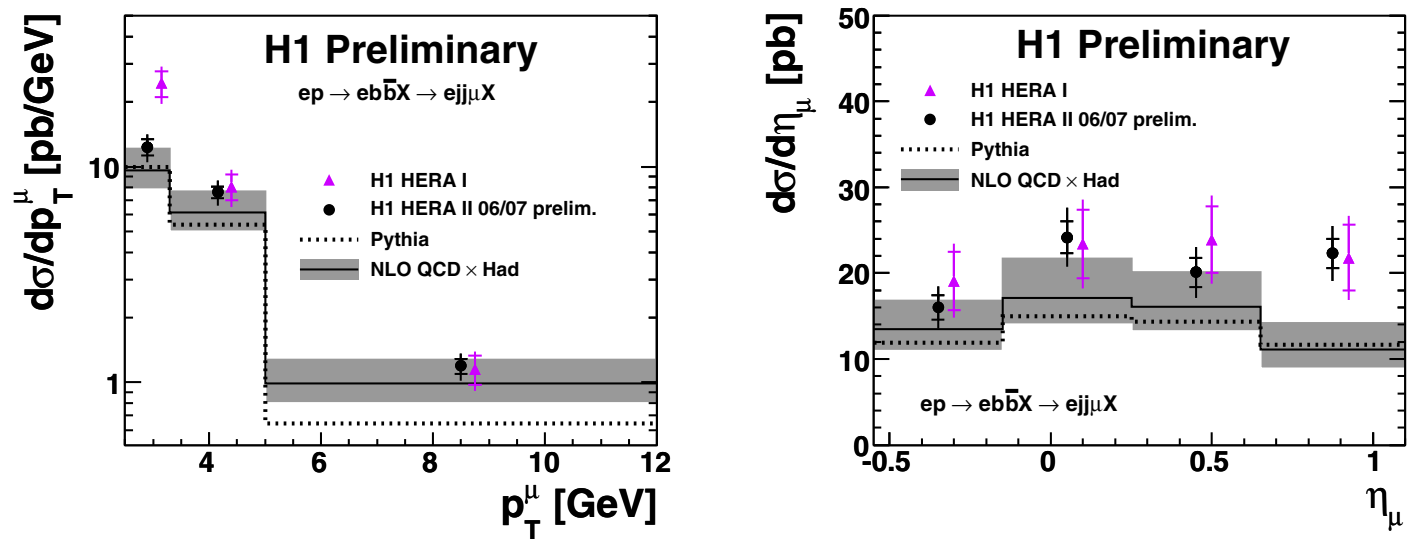

Fig. 9. Differential cross sections as a function of $p_{\mathrm{T}}^{\mu}$ and $\eta^{\mu}$. 


\subsection{Beauty in dijet photoproduction using in- clusive secondary vertexing}

A dataset corresponding to an integrated luminosity of $128 \mathrm{pb}^{-1}$ was used in this analysis performed by the ZEUS collaboration [18] in photoproduction regime. The events containing at least 2 jets with $p_{\mathrm{T}}^{\text {jet1(2) }}>7(6) \mathrm{GeV}$ were selected.

Exploiting the long lifetime of the B hadrons, a secondary vertexing technique was used to separate the beauty from charm and light flavors, in which secondary vertices were fitted from wellreconstructed tracks associated to jets. Subsequently the 2-dimensional decay length, $d$, was calculated as the distance in $x-y$ between the secondary vertex and the beam spot, which was then projected onto the jet axis. In order to further improve the separation between signal and background, the decay length, $d$, was combined with the invariant mass, $m_{\mathrm{vtx}}$, of the tracks fitted to the corresponding secondary vertex. The beauty contribution was then determined from a $\chi^{2}$-fit of the beauty, charm and light flavor MC distributions to the data, simultaneously performed in all three $m_{\mathrm{vtx}}$ bins.

Differential cross sections as a function of $p_{\mathrm{T}}^{\text {jet }}$ and $\eta^{\text {jet }}$ were measured and compared to the scaled PYTHIA MC predictions as well as to NLO QCD predictions calculated with the FMNR programme. Good agreement between the measurements and both predictions was observed.
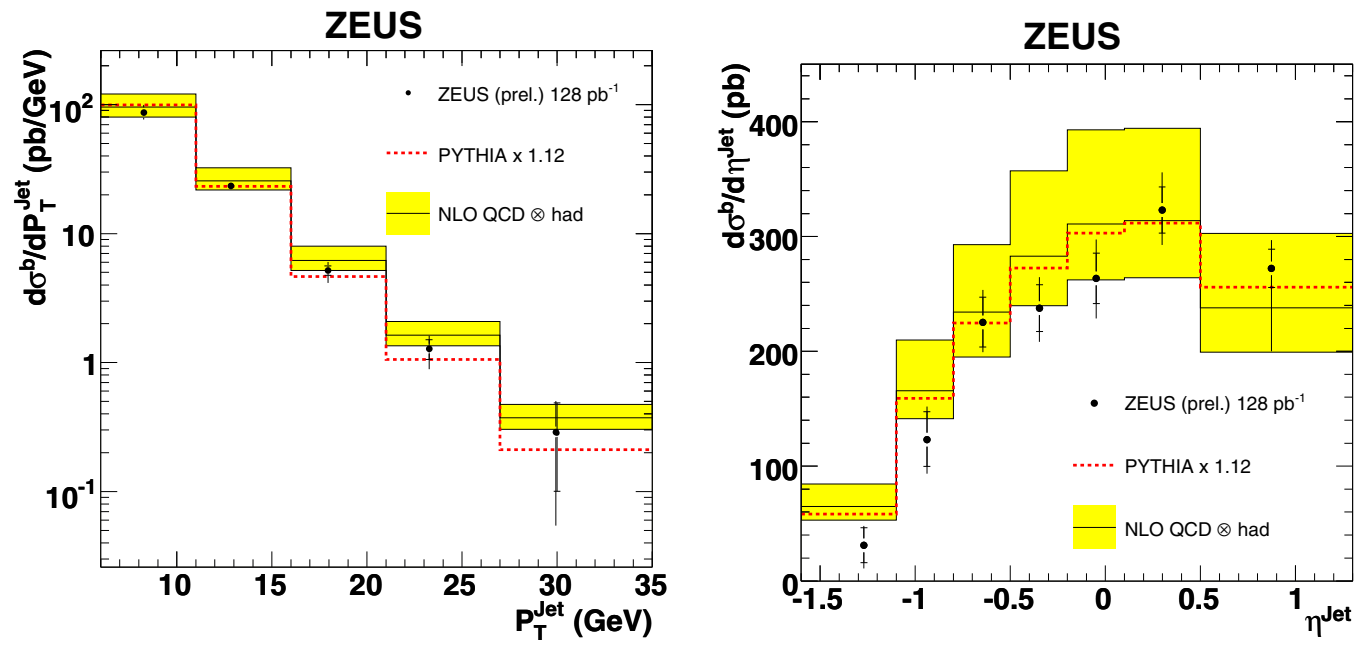

Fig. 10. Differential cross sections as a function of $p_{\mathrm{T}}^{\text {jet }}$ and $\eta^{\text {jet }}$.

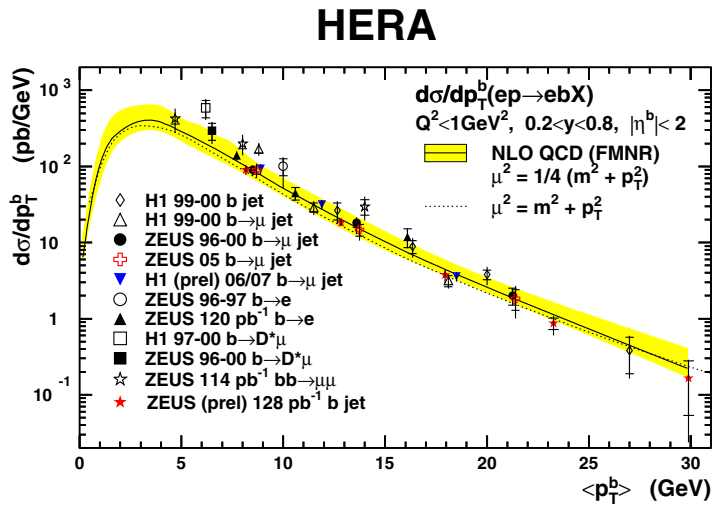

Fig. 11. Cross sections for beauty production a function of $p_{\mathrm{T}}^{\mathrm{b}}$ from various $\mathrm{H} 1$ and ZEUS measurements.

The various $\mathrm{H} 1$ and ZEUS measurements using different experimental techniques are displayed in the summary plot (Fig. 11) as a function of $p_{\mathrm{T}}^{\mathrm{b}}$. The measurements presented in this article denoted by open crosses, downward-pointing triangles, upwardpointing triangles and filled stars in Fig. 11, agree well with all previous measurements. The NLO QCD calculattion provides a good description of data over a wide range of $p_{\mathrm{T}}^{\mathrm{b}}$.

\section{$5 \quad$ Summary}

The production cross sections of the strange mesons, $\mathrm{K}_{\mathrm{S}}^{0}, \Lambda, \mathrm{K}^{* \pm}, \mathrm{K}^{* 0}$ and $\phi$ are measured inclusively. Meanwhile the differential cross sections as a function of kinematic variables are also measured, which is in reasonable agreement with the predictions of leading order MC predictions. The $\Lambda-\bar{\Lambda}$ asymmetry is found to be consistent with zero. The inclusive $\mathrm{K}_{\mathrm{S}}^{0} \mathrm{~K}_{\mathrm{S}}^{0}$ mass spectrum is investigated and three clear 
peaks corresponding to $\mathrm{f}_{2}(1270) / \mathrm{a}_{2}^{0}(1320), \mathrm{f}_{2}^{\prime}(1525)$ and $f_{0}(1710)$ are observed. The widths and masses obtained from two different fits are consistent with the PDG values.

The inclusive production of $\mathrm{D}^{*}$ mesons in phtoproduction and the corresponding differential cross sections as a function of different kinematic variables are presented. The comparison to the NLO QCD calculation given by the FMNR programme shows that the NLO QCD calculation provides a reasonable description of data. The excited charm and charmstrange mesons were studied in both photoproduction

\section{References}

1 Kopeliovich B, Povh B. Z. Phys. C, 1997, 75: 693-699

2 ZEUS collaboration, Holm U (ed.) The ZEUS Detector. Status Report (unpublished), DES, 1993, available on http://www-zeus.desy.de/bluebook/bluebook.html; Abt I et al (H1 collaboration). Nucl. Instrum. Meth. A, 1997, 386: $310-347$

3 Aaron F D et al (H1 collaboration). Eur. Phys. J. C, 2009, 61: 185-205; Aaron F D et al (H1 collaboration). H1prelim$08-132$

4 Aaron F D et al (H1 collaboration). Phys. Lett. B, 2009, 673: $119-126$

5 Chekanov S et al (ZEUS collaboration). Phys. Rev. Lett., 2008, 101: 112003

6 Amsler C et al. Phys. Lett. B, 2008, 667: 1-1340

7 Aaron F D et al (H1 collaboration). H1prelim-08-073

8 Chekanov S et al (ZEUS collaboration). Eur. Phys. J., 2009, and DIS regime. The fragmentation rates of c quarks hadronising as a given excited charm meson are measured, which are consistent with previous measurements.

The beauty production was measured using three different experimental techniques. The results are in good agreement with previous measurements. The measured cross sections as a function of $p_{\mathrm{T}}^{\mathrm{b}}$ are compared to the NLO QCD calculation given in FMNR programme. A general agreement with the NLO prediction is observed, giving a consistent picture of $\mathrm{b}$ quark photoproduction over a wide range of $p_{\mathrm{T}}^{\mathrm{b}}$.

60: $25-45$

9 Ackerstaff K et al (OPAL collaboration). Z. Phys. C, 1997, 76: $425-440$

10 Heister A et al (ALEPH collaboration). Phys. Lett. B, 2002, 526: $34-49$

11 Becattini F. Z. Phys. C, 1996, 69: 485-492

12 Abbiendi G et al. Eur. Phys. J. 2001, 20: 445-454

13 Chekanov S et al (ZEUS collaboration). Phys. Rev. D, 2008, 78: 072001

14 Chekanov S et al (ZEUS collaboration). arXiv:0901.2226 [hep-ex]

15 Aaron F D et al (H1 collaboration). H1prelim-08-071

16 Aktas A et al (H1 collaboration). Eur. Phys. J. C, 2005, 41: $453-467$

17 Chekanov S et al (ZEUS collaboration). Phys. Rev. D, 2004, 70: 12008; Phys. Rev. D, 2006, 74: 059906

18 Chekanov $\mathrm{S}$ et al (ZEUS collaboration). ZEUS-prel-09-005 